\title{
The Effect of Deadtime and Pulse Processing Time Constant on Standards-based Quantitative EDS Microanalysis
}

\author{
Stephen Seddio
}

Thermo Fisher Scientific, Fitchburg, Wisconsin, United States

EDS (energy-dispersive X-ray spectroscopy) is now, by far, the most commonly used method of X-ray microanalysis. Accurate and precise quantitative analysis is possible. However, the rigor needed (e.g., the use of standards, proper sample preparation, etc.) to obtain accurate and precise results is often ignored. Even when standards-based EDS quantitative microanalysis is done, some guidelines are commonly overlooked. For example, a detector deadtime on $10 \%$ or less is suggested in order to minimize sum peaks [1]. However, such low deadtimes can require impractically long acquisitions times, particularly when analyzing for minor or trace elements.

Ideally, EDS spectra acquired for peak reference shapes and/or peak intensities should be acquired with the same conditions (e.g., accelerating voltage, working distance, deadtime, and pulse processing time constant) as "unknown" spectra. However, an unintended, yet possibly common, practice would be to acquire spectra from standard sampless with great rigor, and then acquire unknown spectra with less rigor. Here, I explore the effect from two such deviations:

-Acquiring standard spectra with a 10\% deadtime and unknown spectra with a higher deadtime

-Acquiring standard spectra with a slow pulse processing time constant (lower throughput) and unknown spectra with a faster pulse processing time constant (higher throughput).

The samples used for this work were on a mineral standards block commercially available from Structure Probe, Inc. A Cr-diopside standard and a quartz standard were used for the acquisition of unknown spectra. For the analysis of the $\mathrm{Cr}$-Diopside, $\mathrm{Mg}, \mathrm{Si}, \mathrm{Ca}$, and $\mathrm{Fe}$ were quantified, and all other elements were declared to have the published composition for the matrix correction. For the analysis of quartz, Si was quantified, and $\mathrm{O}$ was declared to have the published composition for the matrix correction. All elements were quantified using their K-lines, and standard peak reference shapes and intensities were taken from the same spectra. The $\mathrm{Mg}$ and Fe K-lines were standardized using olivine, the Si K-lines were standardized using quartz, and the $\mathrm{Ca} \mathrm{K}$-lines were standardized using (non-Cr) diopside.

The Samples were analyzed in a JEOL JSM-7001F SEM. EDS data was acquired using a windowless 10 $\mathrm{mm}^{2}$ active area Thermo Scientific ${ }^{\mathrm{TM}}$ UltraDry $^{\mathrm{TM}}$ EDS detector and processed using the Thermo Scientific $^{\mathrm{TM}}$ Pathfinder ${ }^{\mathrm{TM}} \mathrm{X}$-ray Microanalysis system. The beam current was measured using an incolumn Faraday cup at the beginning of each acquisition. Additional processing using the "derivative references" option in Pathfinder, was not done. Sum and escape peaks were removed from acquired standard and unknown spectra and those counts were added to the peaks to which they belonged.

For the analysis of $\mathrm{Si}$ in quartz and the analysis of $\mathrm{Mg}, \mathrm{Si}, \mathrm{Ca}$, and $\mathrm{Fe}$ in $\mathrm{Cr}$-Diopside, the calculated concentrations of all elements are closest to the published compositions when the deadtime used to acquire the unknown spectra was kept below 30\%, which is similar to the deadtime used to acquire the standard spectra. Similarly, quantifying $\mathrm{Si}$ in quartz yielded the best results when the unknown spectra were acquired using a time comstant similar to the time constant used for acquiring the standard spectra (6400 ns). 
When elements present at major concentrations $(\mathrm{Mg}, \mathrm{Si}$, and $\mathrm{Ca})$ were quantified using high deadtimes or inconsistent time constants the results were within $3 \mathrm{wt} \%$ of the expected results. Therefore, if quantitative accuracy is the goal of an analysis, then care should be taken to ensure that unknown and standard spectra are acquired with low and similar deadtimes and with identical time constants. However, if the goal of the EDS quantitative analysis is to determine matrix correction factors for trace elements (something that would be appropriate for combined EDS and WDS quantitative mapping), using higher deadtimes and different time constants will likely not have a dramatic effect on the results.
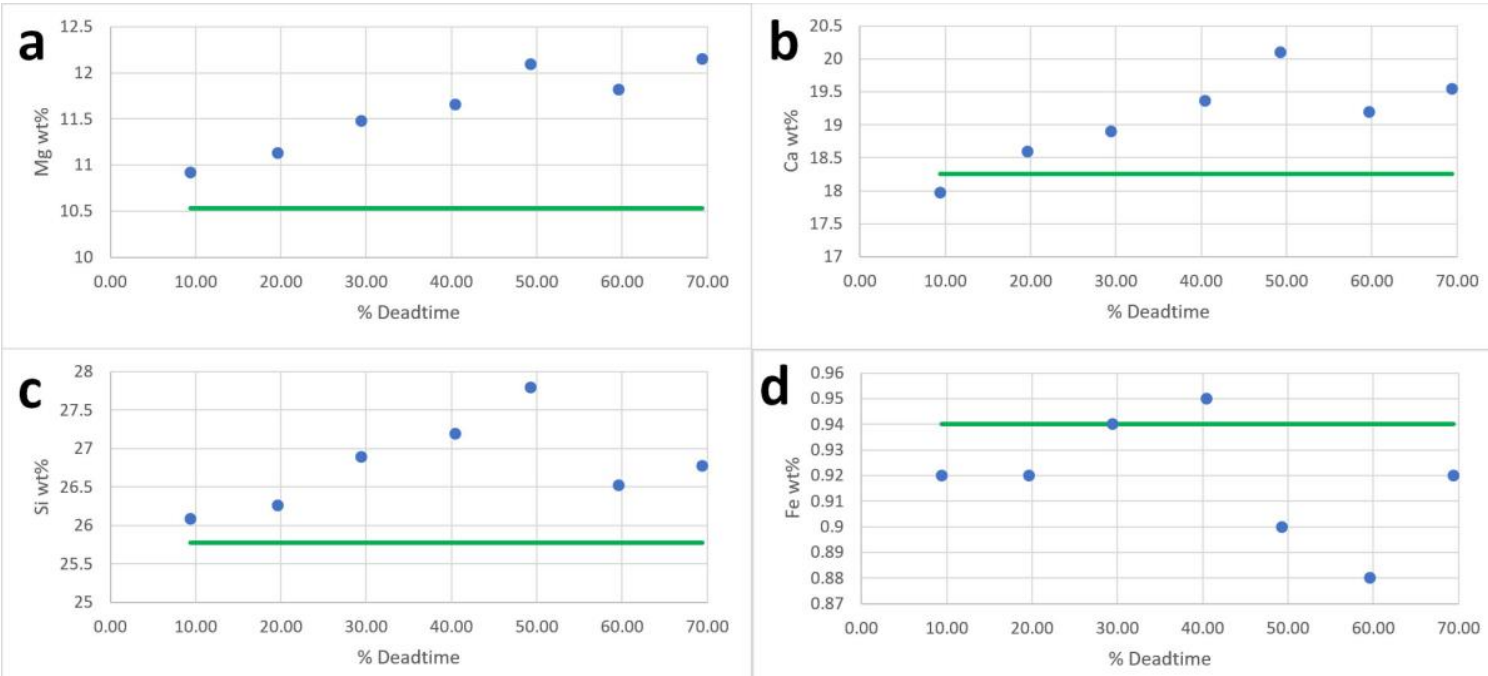

Figure 1. Plots of calculated wt\% vs. deadtime for the four quantified elements in Cr-Diopside. The horizontal green line represents the published concentrations.
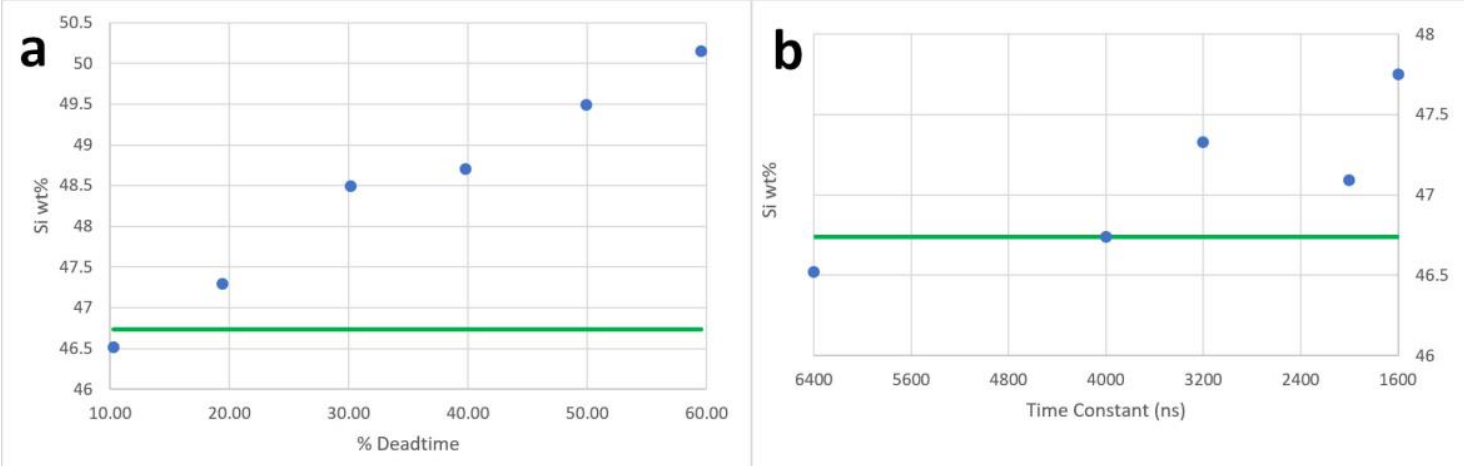

Figure 2. Figue $2 \mathrm{a}$. A plot of wt $\% \mathrm{Si}$ vs. deadtime from the analysis of quartz. Figure $2 \mathrm{~b}$. A plot of wt $\%$ $\mathrm{Si}$ vs. time constant from the analysis of quartz. The horizontal green line represents the published concentration.

References

[1] DE Newbury and NWM Ritchie, Journal of Materials Science 50 (2015), p. 493. 